\title{
Afterglows of gamma-ray bursts
}

Shrinivas R. Kulkarni, Edo Berger, Joshua Simon Bloom, Frederic H. Chaffee, Alan H. Diercks, et al.

Shrinivas R. Kulkarni, Edo Berger, Joshua Simon Bloom, Frederic H. Chaffee, Alan H. Diercks, S. George Djorgovski, Dale A. Frail, Titus J. Galama, Robert Goodrich, Fiona A. Harrison, Re'em Sari, S. A. Yost, "Afterglows of gamma-ray bursts," Proc. SPIE 4005, Discoveries and Research Prospects from 8- to 10-Meter-Class Telescopes, (29 June 2000); doi: $10.1117 / 12.390135$ 


\title{
The afterglows of gamma-ray bursts
}

\author{
S. R. Kulkarni*, E. Berger*, J. S. Bloom*, F. Chaffee", \\ A. Diercks*, S. G. Djorgovski*, D. A. Frail ${ }^{\dagger}$, T. J. Galama*, R. W. Goodrich \\ F. A. Harrison*, R. Sari* \& S. A. Yost* \\ "California Institute of Technology, Pasadena, CA 91125, USA \\ † National Radio Astronomy Observatory, Socorro, NM 87801, USA \\ IW. M. Keck Observatory, Kamuela, HI 96743, USA
}

\begin{abstract}
Gamma-ray burst astronomy has undergone a revolution in the last three years, spurred by the discovery of fading long-wavelength counterparts. We now know that at least the long duration GRBs lie at cosmological distances with estimated electromagnetic energy release of $10^{51}-10^{53} \mathrm{erg}$, making these the brightest explosions in the Universe. In this article we review the current observational state of the long-lived "afterglow" emission that accompanies GRBs at X-ray, optical, and radio afterglow wavelengths. We then discuss the insights these observations have given to the progenitor population, the energetics of the GRB events, and the physics of the afterglow emission. We focus particular attention on the evidence linking GRBs to the explosion of massive stars. Throughout, we identify remaining puzzles and uncertainties, and emphasize promising observational tools for addressing them. The imminent launch of HETE-2, the increasingly sophisticated and coordinated ground-based and space-based observations, and the increasing availability of 10-m class optical telescopes have primed this field for fantastic growth.
\end{abstract}

\section{INTRODUCTION}

Gamma-ray bursts (GRBs) have mystified and fascinated astronomers since their discovery. Their brilliance and their short time variability clearly suggest a compact object (black hole or neutron star) origin. Three decades of high-energy observations, culminating in the definitive measurements of CGRO/BATSE, determined the spatial distribution to be isotropic yet inhomogeneous, suggestive of an extragalactic population (see Fishman and Meegan ${ }^{1}$ for a review of the situation prior to the launch of the BeppoSAX mission). Further progress had to await the availability of GRB positions adequate for identification of counterparts at other wavelengths.

In the cosmological scenario, GRBs would have energy releases comparable to that of supernovae (SNe). Based on this analogy, Paczyński \& Rhoads ${ }^{2}$ and $\mathrm{Katz}^{3}$ predicted that the gamma-ray burst would be followed by long-lived but fading emission. These papers motivated systematic searches for radio afterglow, including our effort at the VLA. ${ }^{4}$ The broad-band nature of this "afterglow" and its detectability was underscored in later work. ${ }^{5,6}$

Ultimately, the detection of the predicted afterglow had to await localizations provided by the Italian-Dutch satellite, BeppoSAX. ${ }^{7}$ The BeppoSAX Wide Field Camera (WFC) observes about $3 \%$ of the sky, triggering on the low-energy $(2-30 \mathrm{keV})$ portion of the GRB spectrum, localizing events to $\sim 5-10$ arcminutes. X-ray afterglow was first discovered by BeppoSAX in GRB 970228, after the satellite was re-oriented (within about 8 hours) to study the error circle of a WFC detection with the $2-10 \mathrm{keV} \mathrm{X-ray} \mathrm{concentrators.} \mathrm{The} \mathrm{detection} \mathrm{of} \mathrm{fading} \mathrm{X-ray} \mathrm{emission,}$ combined with the high sensitivity and the ability of the concentrators to refine the position to the arcminute level, led to the subsequent discovery of long-lived emission at lower frequencies ${ }^{8-10}$ (e.g. Figure 1).

Optical spectroscopy of the afterglow of GRB 970508 carried out at the Keck Observatory led to the definitive demonstration of the extragalactic nature of this GRB. ${ }^{11}$ The precise positions provided by radio and/or optical afterglow observations have allowed for the identification of host galaxies, found in almost every case. Not only has this provided further redshift determinations, but it has been useful in tying GRBs to star formation through measurements of the host star formation rate (e.g. ${ }^{12,13}$ ). HST with its exquisite resolution has been critical in localizing GRBs within their host galaxies and thereby shed light on their progenitors (e.g. ${ }^{14-16}$ ). Observations of the radio afterglow have directly established the relativistic nature of the GRB explosions ${ }^{10}$ and provided evidence

Further author information: (Send correspondence to S.R.K.)

S.R.K.: E-mail: srk@astro.caltech.edu 
linking GRBs to dusty star-forming regions. Radio observations are excellent probes of the circumburst medium and the current evidence suggests that the progenitors are massive stars with copious stellar winds. The latest twist is an apparent connection of GRBs with SNe. ${ }^{17}$ Separately, an important development is the possible association of a GRB with a nearby $(40 \mathrm{Mpc})$ peculiar SN. ${ }^{18,19}$
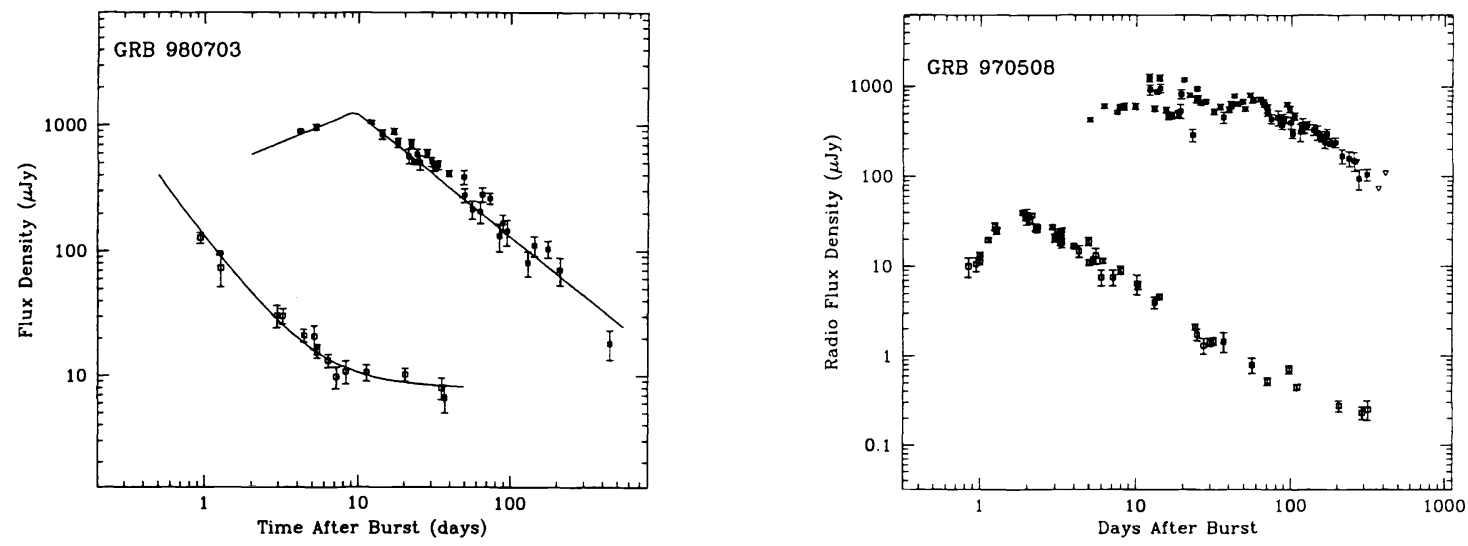

Figure 1. The radio (filled squares) and optical (open squares) light curves of GRB 980703 (left) and GRB 970508 (right). These are typical afterglow. The radio shows a rise to maximum, followed by a power law decay. One expects a similar lightcurve at optical and X-ray bands except that the peak flux is achieved on very short timescales, tens of seconds to tens of minutes. However, most optical observations are conducted on longer timescales and only the power law decay is seen. The flattening of the optical emission seen in the afterglow of GRB 980703 is due to the contribution from the underlying host galaxy. The host galaxy of GRB 970508 is considerabley fainter and the flattening is less obvious.

In this paper we review the primary advances resulting from afterglow studies. In the previous review ${ }^{20}$ we emphasized the observations of radio afterglow. Here the emphasis is on optical afterglow. In $\S 2$ we review constraints on the nature of the progenitor population(s), in particular evidence linking some classes of GRBs to SNe. §3 describes the status of current understanding of the physics of the afterglow emission. Here we compare observations to predictions of the basic spherically-symmetric model, and describe complications arising from deviations from spherical symmetry and non-uniform distribution of the circumburst medium.

\section{THE NATURE OF THE PROGENITORS}

In almost all cases, a host galaxy has been identified at the location of the fading afterglow (see Figs. 2 and 5 ). GRB redshifts can be obtained either via absorption spectroscopy (when the transient is bright) or by emission spectroscopy of the host galaxy (Figure 4). In Figure 3 and Table 1 we summarize the measured redshifts and host galaxy magnitudes. While the distance scale debate is settled (at least for the class of long duration GRBs, see below) it is difficult to overstress the importance of continued redshift determination. Not only do redshifts afford a robust determination of the energetics (Figure 3), a redshift to a GRB also sets a physical scale to observed angular offsets as well as allows a determination of the nature of the host galaxy (size, star-formation, etc.).

Despite the advances, we remain relatively ignorant of the nature of the central engine. Currently popular GRB models fall into two categories: (i) the coalescence of compact objects (neutron stars, black holes and white dwarfs ${ }^{24-27}$ ) and (ii) the collapse of the central iron core of a massive star to a spinning black hole, a "collapsar" ${ }^{28}$ We now summarize the light shed on the progenitor problem by afterglow studies.

The Location of GRBs Within Hosts. A fundamental insight into the nature of SNe came from their location with respect to other objects within the host galaxy (specifically HII regions and spiral arms) and the morphology of the host galaxy itself (elliptical versus spiral). In a similar manner, we are now making progress in understanding 
GRB 990123
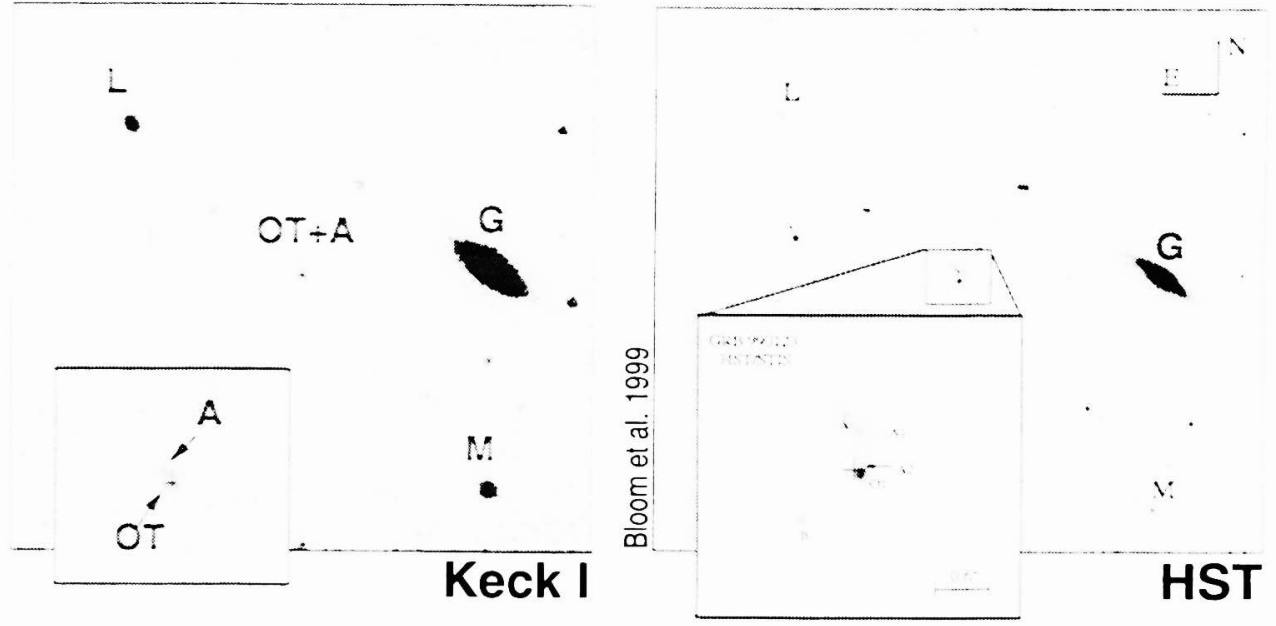

Figure 2. Deep Keck $K$-band and Hubble Space Telescope (HST) optical images of the afterglow of GRB 99012:3 and its host galaxy. In both the groundbased IR image and the HST image, both taken about three weeks after the GRB. the afterglou is. clearly resolved from its host. The 1- $\sigma$ ( 100 milliarcsec) position of the early transient from ground-based image is denoted with cross-hairs on the HST inset: the OT is clearly offset from the centroid light of the host and thus not likely to be the resuit of any $A G N$ activity. It appears that the host is a disturbed. perhaps interacting system.

Table 1. Basic Properties of Selected GRBs

\begin{tabular}{|c|c|c|c|c|c|c|}
\hline GRB & $\begin{array}{c}\alpha(\mathrm{J} 2000) \\
(\mathrm{h} \text { II s) }\end{array}$ & $\begin{array}{c}\delta(\mathrm{J} 2000) \\
\left(0,{ }^{\prime \prime}\right)\end{array}$ & $\begin{array}{l}\mathrm{R}_{\text {host }} \\
\text { (mag) }\end{array}$ & $\begin{array}{c}\mathrm{S} \times 10^{-6} \\
\left(\mathrm{erg} \mathrm{cm}^{-2}\right.\end{array}$ & $z$ & References $^{\dagger}$ \\
\hline 970228 & 050147 & +1146.9 & $25.2^{\star}$ & 1.7 & 0.695 & Djorgorski et al. GCN 289 \\
\hline 970508 & 065349 & +7916.3 & 25.7 & 3.1 & 0.835 & 11,21 \\
\hline 970828 & 180832 & +591852 & 24.5 & 74 & 0.957 & 22 \\
\hline 971214 & 115626 & +6512.0 & 25.6 & 11 & 3.418 & 12 \\
\hline 980326 & 083634 & -1851.4 & 227.3 & 1 & & \\
\hline 980329 & 070238 & +3850.7 & 25.4 & 50 & & \\
\hline 980519 & 212221 & +7715.7 & 26.2 & 25 & & \\
\hline 980613 & 101758 & +7127.4 & 24.5 & 1.7 & 1.096 & Djorgouski et al. GCN 189 \\
\hline 980703 & 235907 & +0835.1 & 22.6 & 37 & 0.966 & \\
\hline 981226 & 232937 & -235554 & $\geq 22$ & NA. & 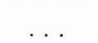 & \\
\hline 990123 & 152531 & +444600 & 24.4 & 265 & 1.600 & 23 \\
\hline 990510 & 133807 & -802949 & 228 & 23 & 1.619 & Vreeswijk et al. GCN 324 \\
\hline 990712 & 223153 & -732429 & 21.78 & N.A. & 0.430 & Galama et al. GCN 388 \\
\hline 991208 & 163354 & +462721 & $\geq 25$ & 100 & 0.706 & Dodonov et al. GCN 475 \\
\hline 991216 & 050931 & +111707 & 24.5 & 256 & 1.020 & Vreeswijk et al. GCN 496 \\
\hline
\end{tabular}

+ References to redshift determination.

- $V^{-}$-band magnitude from HST. All others are R magnitude in the Johnson system.

GRB progenitors by measuring offsets with respect to other objects in the host galaxies. The rather good coincidence of GRBs with host galaxies already suggests that they are unlikely to be a halo population (as would be expected in the coalescence scenario ${ }^{29}$ ). On the other hand. with the possible exception of GRB $970508 .{ }^{30}$ they are clearly not associated with galactic nuclei (i.e. massive central black holes). Typical offsets of GRBs from the centroid of their host galaxies are comparable to the half-light radii of field galaxies at comparable magnitudes. suggesting that GRBs originate from stellar populations.

Host Galaxies. Demonstrating a direct link between GRBs and (massive) star formation is more difficult. In both popular progenitor models. the rate of GRBs is expected to closely follow the star formation rate of Universe 


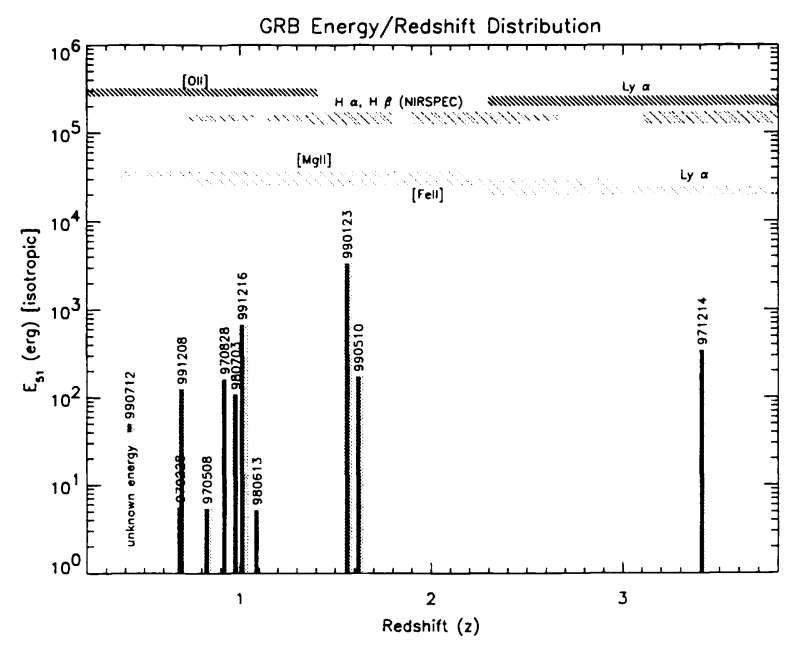

Figure 3. The isotropic gamma-ray energy distribution of GRBs with confirmed redshifts. Bursts indicated in black are those with spectroscopically confirmed emission lines from the host galaxies; bursts indicated by a shaded column (e.g. 990123) are those with absorption line redshifts. The relevant key absorption or emission features are noted at the top of the figure.

and so we expect that that all GRBs should be associated with star forming galaxies (e.g. irregulars, late-type). The discovery of a GRB associated with an early-type without significant star formation would be difficult to accommodate. On the whole, the population of identified hosts seems typical in comparison to irregular and latetype field galaxies in the same redshift and magnitude range (see Fig. 5 and table 1). The hosts have average luminosities for field galaxies, modulo corrections due to evolution. Their emission line fluxes and equivalent widths are also statistically indistinguishable from the normal field galaxy population. The observed star formation rates, derived from recombination line fluxes (mostly the from the [O II] $3727 \AA$ and UV continuum flux) are $M_{\odot} \mathrm{yr}^{-1}$ to several tens of $M_{\odot} \mathrm{yr}^{-1}$ - typical of normal galaxies at comparable redshifts (extinction corrections can increase these numbers by a factor of a few, but similar corrections apply to the comparison field galaxy population as well). It will probably be necessary to have a sample of several tens of GRB hosts before a correlation of GRBs with the (massive) star formation rate can be tested statistically. However, below we point to several specific examples which are suggestive of a link between GRBs and star-forming regions.

Association with Star-forming Regions. There is evidence showing that GRBs arise from dusty regions within their host galaxies. In this respect, radio observations provide a unique tool for detecting events in regions of high ambient density (as was the case for GRB 980329). An even more extreme example is GRB 970828, where the host was identified based solely on the VLA discovery of a radio flare. ${ }^{22}$ Interestingly enough, this is the dustiest galaxy in the sample of GRB hosts to-date.

Second, some GRBs appear to be located within identifiable star-forming regions. An example is GRB 990123 in Figure 2. ${ }^{16,32,15}$ VLA observations of GRB $980703^{33}$ are perhaps more convincing. The radio observations can be sensibly interpreted by appealing to free-free absorption from a foreground HII region (which would dwarf the Orion complex). If this interpretation is correct then this would be strong evidence for a GRB being located within a starburst region.

The GRB-SN link. If GRBs arise from the collapse of a massive star, it is an unavoidable consequence that emission from the underlying supernova should be superimposed on the afterglow. Bloom et al. ${ }^{17}$ may have made the first detection of a possible SN component in the GRB 980326 lightcurve (Figure 6). These authors noted that $\mathrm{SNe}$, in contrast to afterglows, have distinctive temporal and spectral signatures: rising to a maximum at $\sim 20(1+z)$ days, with little emission blueward of about $4000 \AA$ in the restframe (and certainly blueward of $3000 \AA$ ) owing to a multitude of resonance absorption lines. This discovery has led to other possible SN detections, most notably GRB $970228 .{ }^{34,35}$ 
The suggestion of a GRB-SN connection is an intriguing one but it has yet to be placed on a firm footing. Important questions are: (i) are all long-duration GRBs accompanied by SNe? (ii) if so, are these SNe of type Ib/c? Ground-based observations are possible in those cases where the afterglow decays rapidly (e.g. GRB 980326) or if high quality optical and IR observations exist (e.g. GRB 970228).

We need more examples to test the GRB-SN link. Future progress will depend on a combination of ground and HST observations. For relatively nearby GRBs especially those with a rapidly decaying optical afterglow it would be attractive and feasible to obtain the spectrum of the SN around the time when the flux from the SN peaks. A moderate quality spectrum with SN-like features would have the singular advantage of definitively confirming the SN interpretation (as opposed to alternatives involving re-radiation by dust $^{36}$ ). However, for most GRBs, we expect HST observations to play a critical role. HST's widely recognized strengths in accurate photometry of sources embedded in galaxies ${ }^{37}$ and photometric stability make the detection of a faint SN against the optical afterglow and the host galaxy possible.

Diversity of the Progenitor Population. As was the case with $\mathrm{SNe}$, it is likely naive to think of a single progenitor population. Below, we discuss the two additional classes which show some promise: the mysterious short duration GRBs and a possible class of low luminosity GRBs associated with SNe.

Short Events. It has been known for some time that the distribution of the duration of GRBs appears to be bimodal. ${ }^{1}$ Furthermore, these two groups may have different spatial distributions, ${ }^{38}$ with the short bursts being detected out to smaller limiting redshifts. However, we know very little about this class of GRBs since, as noted earlier, all bursts localized by BeppoSAX and RXTE thus far are of long duration. Fortunately, improvements in BeppoSAX and the imminent launch of HETE-2 provide for the first time the opportunity to follow-up short GRBs.
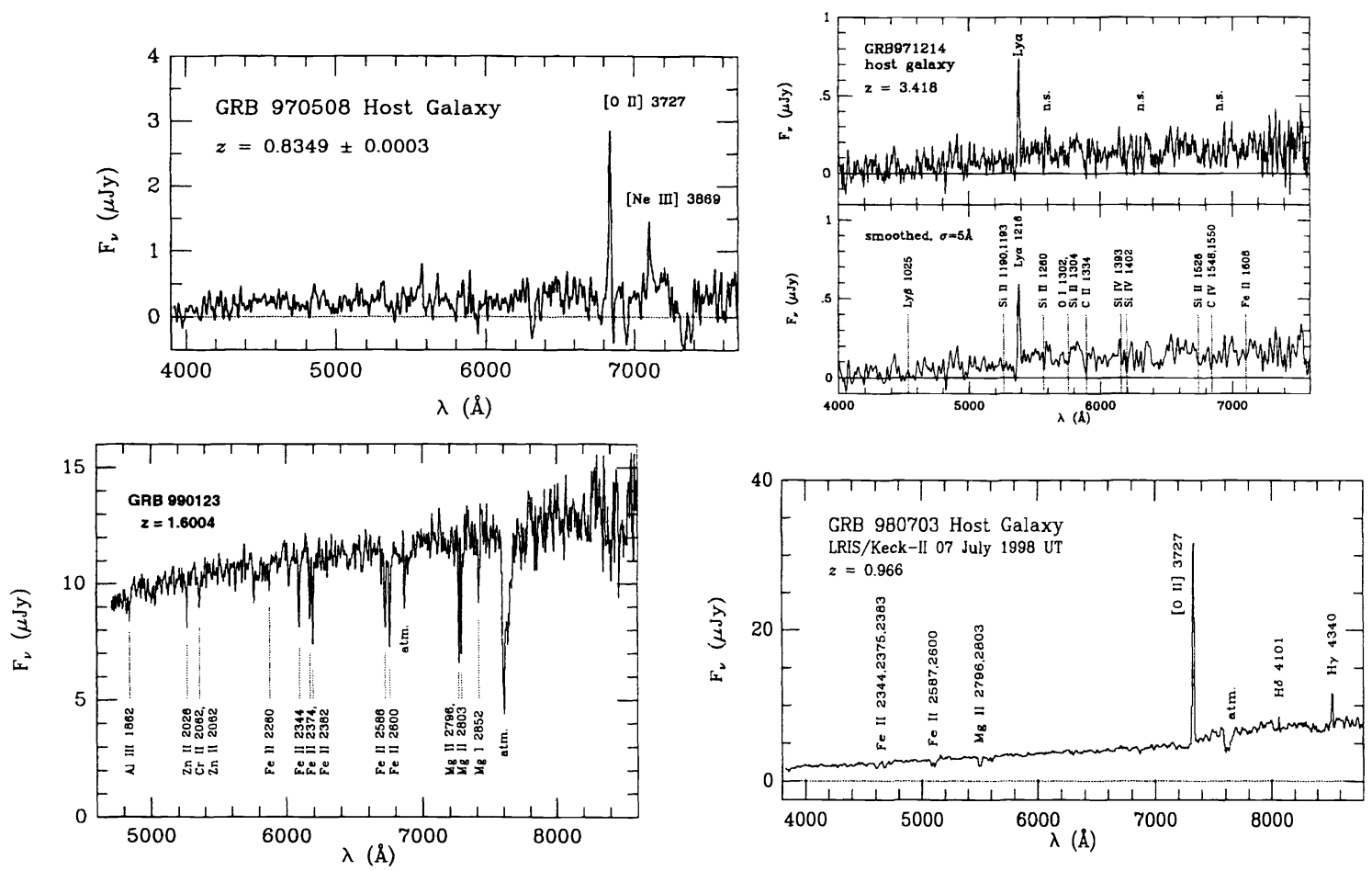

Figure 4. Keck II 10-m spectra of GRBs and their host galaxies. (top left) The spectrum of the host of GRB 970508, the intrinsically faintest galaxy of those detected to date. The [OII] emission line is at the same redshift the systemic velocity of the furthest metal-line absorption system seen when the optical transient was bright (from ref. ${ }^{21}$ ). (top right) The redshifted UV spectrum of the highest redshift host galaxy, GRB 971214. The most prominent line is Ly- $\alpha$ and the spectrum is typical of L* galaxies at comparable redshifts (from ref..$^{12}$ ). (bottom left) The metal-line absorption spectrum through the light of the optical transient of GRB 990123, the brightest GRB to date (from ref. ${ }^{23}$ ). (bottom right) Absorption- and emission-line spectrum of the afterglow and host of GRB 980703, the brightest host galaxy to date (from ref. ${ }^{31}$ ). 


\section{The Host Galaxies of Gamma-Ray Bursts}

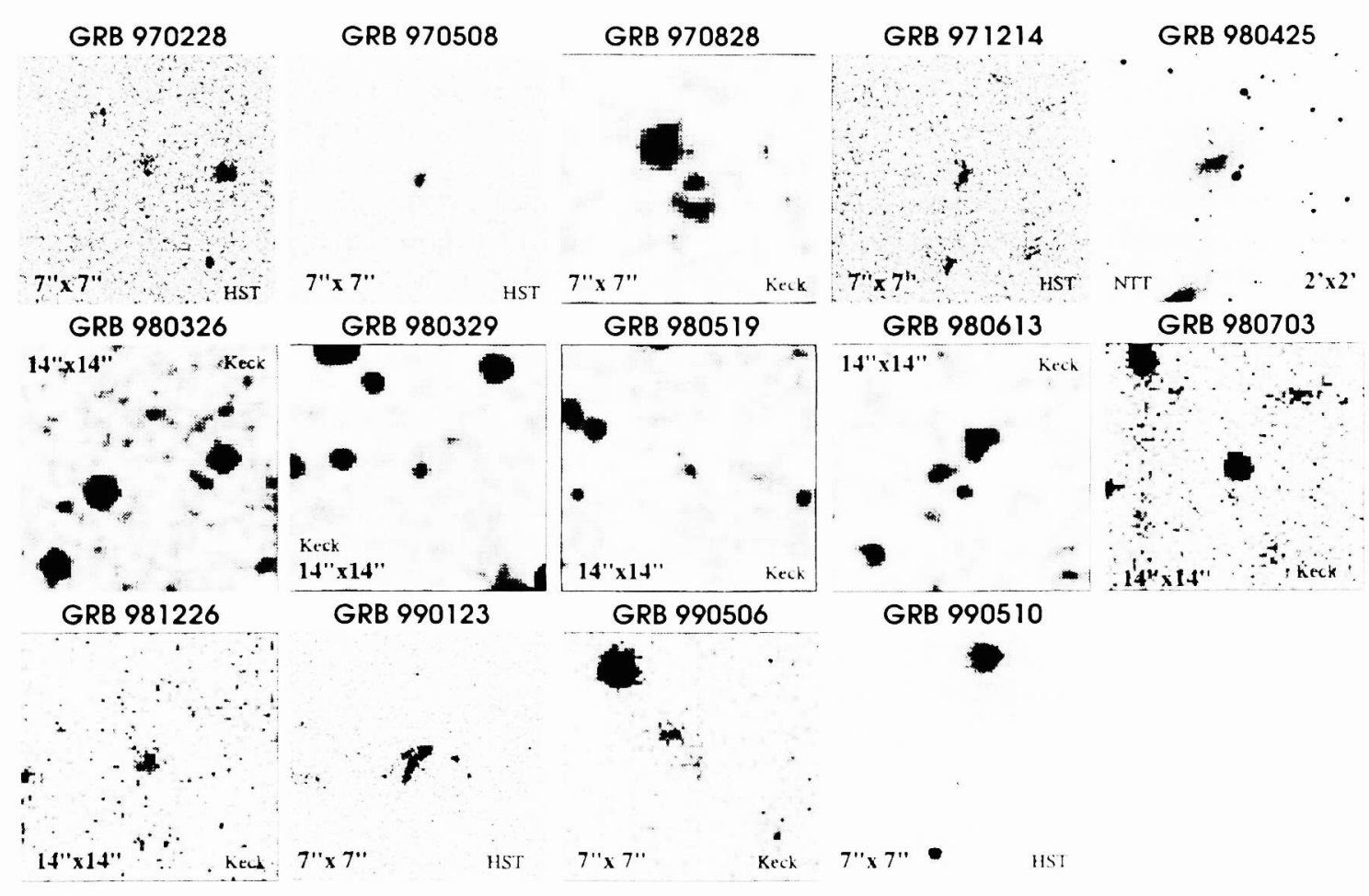

Figure 5. Optical images of GRB hosts from Keck. HST. and the NTT. With the exception of GRB 980326 and GRB 930510. all GRB aftergiow well-localzzed in the optical or radio are astrometrically conncudent near or withen the half-light radzus of a faint (see table 1) gaiary. The best-fit transient position is near the center of each image. All zmages from the heek 10-m are in $R$-band and. excepting GRB 970828. all Heck images are $14 \times 14$ arcsec $^{2}$

The short duration bursts are difficult to accommodate in the collapsar model given the long collapse time of the core. However, they find a natural explanation in the coalescence models. How would these bursts manifest themselves? Li \& Paczynski ${ }^{39}$ speculate that if the short-duration bursts result from NS NS mergers then they may leave a bright. but short-lived $(\lesssim 1$ day) optical transient. Radio observations provide a complementary tool for determining the nature of the short duration bursts. The low ambient density would result in weak afterglow: (since flux $\propto p^{1 / 2}$ ) which are potentially detectable. Radio observations have additional advantages of a longer lived afterglow. immunity from weather and freedom from the diurnal cycle.

Low Luminosity GRBs? The association of GRB 980425 with the nearby type Is SN 1998 b ${ }^{18.19}$ is controversial. If the two are associated then GRB 980425 would be the weakest GRB. ${ }^{40}$ We refer the reader to our recent review ${ }^{20}$ for a summary of the situation.

However, in our opinion. the main issue here is not whether the SN/GRB association is valid but the unusual nature of SN 1998bw. We have argued that ${ }^{29}$ this S.N has a mildly relativistic shock. carrying as much as $10^{50}$ erg

a feature never seen in any S.N. This relativistic shock is, of course. in addition to the usual sub-relativistic S.M shock. This energy is similar to the energy inferred in cosmologically located GRBs, and suggests that there may well be a continum of GRB energies. Progress in this field will be possible only when other examples are identified. For this reason, our discussion here is necessarily brief.

\section{AFTERGLOW: THE PHYSICS AND ENERGETICS OF THE FIREBALL}

One can consider a GRB to be like a SN explosion with a central source releasing energy $E_{0}$ (comparable to the mechanical release of energy in an SN). This is the so-called fireball model. The difference between an SN and a 


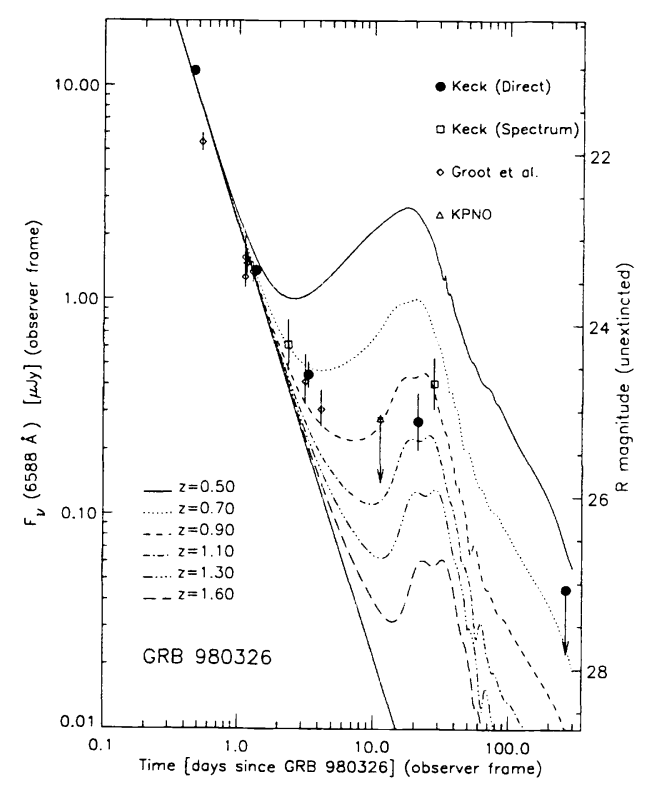

light curves of GRB 970228

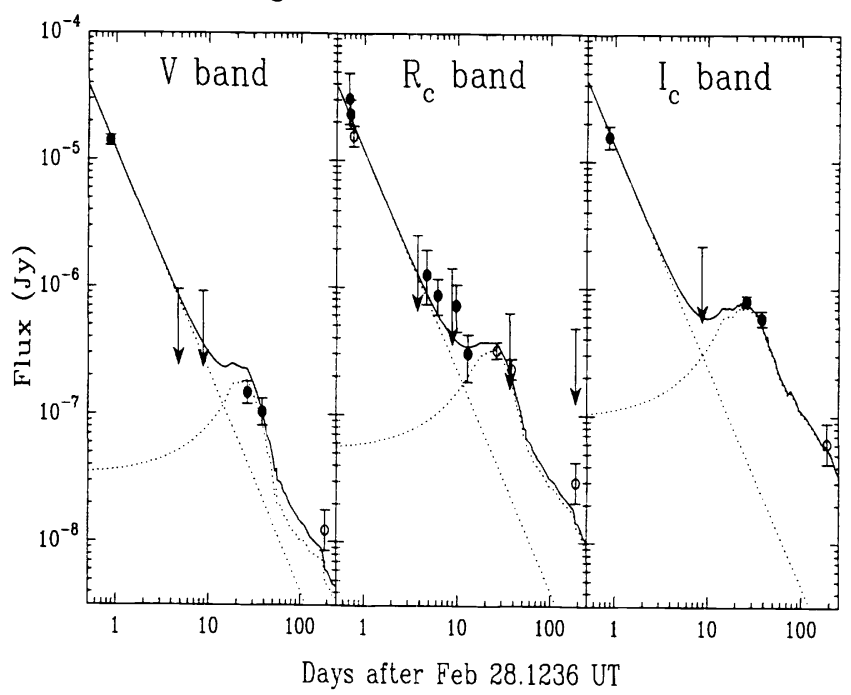

Figure 6. R-band light curve of GRB 980326 (top) and the sum of an initial power-law decay plus Ic supernova light curve for redshifts ranging from $z=0.50$ to $z=1.60$; from reference. ${ }^{17}$ Similarly, the light-curve of the OT of GRB 970228 (bottom). With this burst the redshift is known $(z=0.695)$ and the degeneracy between peak supernova brightness and distance is resolved. The fit to a $S N+O T$ model fits remarkably well ${ }^{34}$ : other than the early-time decay, there are no free parameters.

GRB is primarily in ejecta mass: $1-10 M_{\odot}$ for SNe whereas only $10^{-5} M_{\odot}$ for GRBs. The evolution of a GRB is much faster than that of a SN due to two factors: the ejecta expand relativistically and, thanks to the smaller ejecta mass, the optical depth is considerably smaller.

As the ejecta encounter ambient gas, two shocks are produced: a short-lived reverse shock (traveling through the ejecta) and a long-lived forward shock (propagating into the swept-up ambient gas). Afterglow emission is identified with emission from the forward shock. In order to obtain significant afterglow emission, several conditions are neces- 
sary. (1) Rapid equipartition of electrons with the shocked protons (which hold most of the energy). (2) Acceleration of electrons to a power law spectrum (particle Lorentz factor distribution, $d N / d \gamma \propto \gamma^{-p}$ ). (3) Rapid growth of the magnetic field with energy density in the range of $10^{-2}$ of that of the protons. Under these circumstances, afterglow emission is dominated by synchrotron emission of the accelerated particles. ${ }^{41,6}$ The weakness of this model is the assumption of growth in the magnetic field strength to the high values noted above (R. Blandford, pers. comm.).

The theoretically expected afterglow spectrum is shown in Figure 7. Three key frequencies can be identified: $\nu_{a}$, the synchrotron self-absorption frequency; $\nu_{m}$, the frequency of the electron with a minimum Lorentz factor (corresponding to the thermal energy behind the shock) and $\nu_{c}$, the cooling frequency. Electrons which radiate above $\nu_{c}$ cool on timescales equal to the age of the shock. The evolution of these three frequencies is determined by the hydrodynamical evolution of the shock which in turn is affected by two principal factors: the environment of the GRB and the geometry of the explosion. For an observer at a fixed frequency, it is the temporal evolution of $\nu_{a}, \nu_{m}$ and $\nu_{c}$ that determines the character of the light curve (e.g. Figure 1).

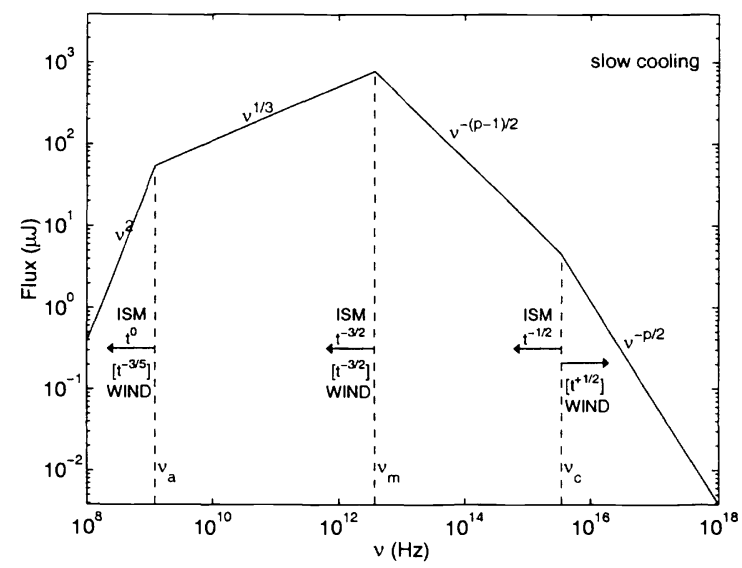

Figure 7. Broad-band spectrum $\left(f_{\nu}\right)$ of the afterglow from a spherical fireball with constant density ("ISM" model; see text) and $\rho \propto r^{-2}$ medium ("wind" model; see text). This is representative of the observed spectrum few days after the burst. Note the distinct evolution of $\nu_{a}$ and $\nu_{c}$ in the two models.

The GRB environment. The earliest afterglow models made the simplifying assumption of expansion into a constant density medium. This is an appropriate assumption should the GRB progenitor explode into a typical location of the host galaxy. However, there is increasing evidence tying GRBs to massive stars (see $\S 2$ ). It is well known that massive stars lose matter throughout their lifetime and thus one expects the circumburst medium to exhibit a density profile, $\rho \propto r^{-2}$ where $r$ is the distance from the progenitor. Chevalier \& $\mathrm{Li}^{42}$ refer to these two models as the ISM (interstellar medium) and the wind model respectively. As can be seen from Figure 7 these two models give rise to rather different evolution of the three critical frequencies.

Geometry: Jets versus Spheres. The hydrodynamics is also affected by the geometry of the explosion. Many powerful astrophysical sources have jet-like structure. There is evidence (from polarization observations) indicating asymmetric expansion in $\mathrm{SNe},{ }^{43}$ so it is only reasonable to assume that GRB afterglows also have jet-like geometry as well. A clear determination of the geometry is essential in order to infer the true energy of the explosion. This is especially important for energetic bursts such as GRB 990123 whose isotropic energy release approaches $M_{\odot} c^{2}$.

Let the opening angle of the jet be $\theta_{0}$. As long as the bulk Lorentz factor, $\Gamma$, is larger than $\theta_{0}^{-1}$, the evolution of the jet is exactly the same as that of a sphere (for an observer situated on the jet axis). However, once $\Gamma$ falls below $\theta_{0}^{-1}$ then two effects become important. First, for a well defined jet, the on-axis observer sees an edge and thus one expects to see a break in the afterglow emission. Second, the lateral expansion of the jet (due to heated and shocked particles) will start affecting the hydrodynamical explosion.

Wind or ISM? The two key diagnostics to distinguish these two models are the evolution of the cooling frequency (see Figure 7) and the early behavior of the radio emission. In the wind model, the radio emission rises rapidly 
(relative to the ISM model) and the synchrotron self-absorption frequency falls rapidly with time. Both these result from the fact that the ambient density decreases with radius (and hence in time) in the wind model.

Unfortunately, in general, the current data are not of sufficient quality to firmly distinguish the two models. For example in GRB 980519, the same optical and X-ray data appear to be adequately explained by the jet+ISM model $^{44}$ and the sphere+wind model. ${ }^{42}$ Including the radio data tips the balance, but only slightly, in favor of the wind model. ${ }^{45}$ In our opinion, the best example for the wind model is that of GRB $980329^{46}$; see Figure 8 . This afterglow exhibits the two unique signatures of the wind model: high $\nu_{a}$ and a rapid rise. Given the importance of making the distinction between the wind and the ISM model we urge early wide band radio observations (especially at high frequencies).
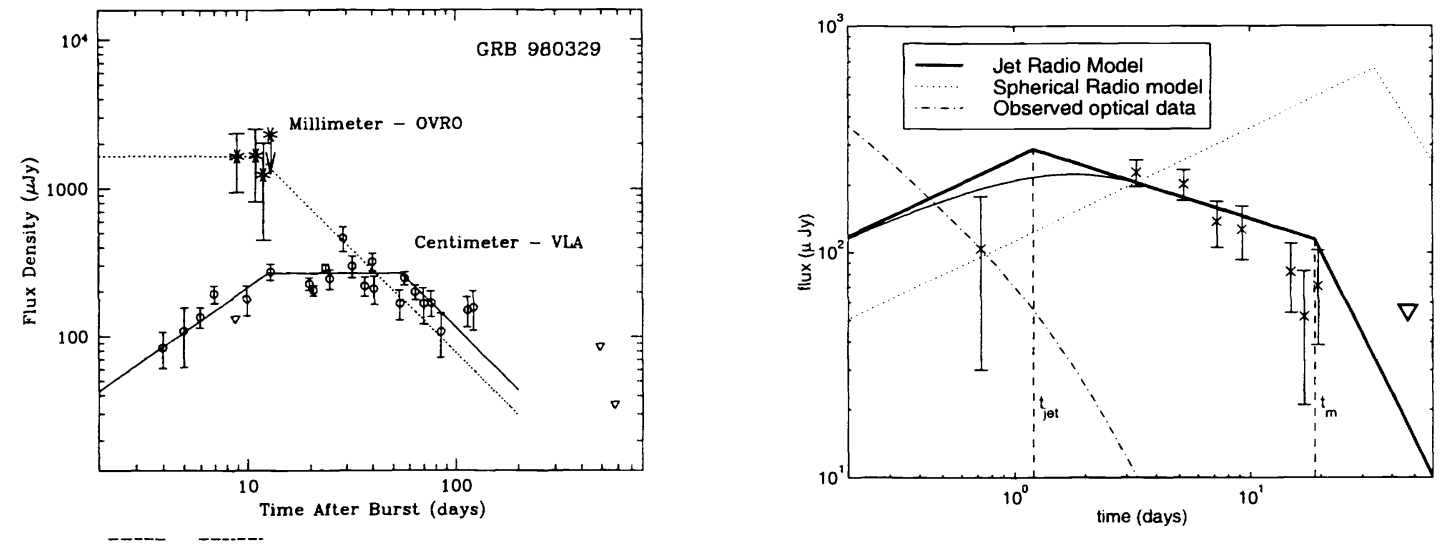

Figure 8. Left: Radio afterglow of GRB 980329. ${ }^{46}$ The rapid rise of the centimeter flux and the high absorption frequency (signified by the considerable strength of the millimeter emission) offer good support for GRB 980329 expanding into a circumburst medium with density falling as inverse square distance. The lines represents a wind model based on X-ray, optical, $I R, \mathrm{~mm}$ and $\mathrm{cm}$ data. Right: Observed and model radio light curves of GRB 990510. ${ }^{47}$ The model predictions for the radio afterglow emission are displayed by the solid line (jet fireball model) and dotted line (spherical fireball model). The observed optical afterglow emission is displayed by the dotted-dashed line; see text for more details.

Energetics. Of all the physical parameters of the fireball, the most eagerly sought parameter is the total energy $E_{0}$. By analogy with supernovae, it is $E_{0}$ which sets the GRB phenomenon apart from other astrophysical phenomena. Classes of GRBs may eventually be distinguished and ranked by their energy budget; for example, long-duration events, short duration events and supernova-GRBs (see $\S 2$ ).

One approach has been to use the isotropic $\gamma$-ray energy as a measure of $E_{0}$; see Figure 3 . There are three well known problems with such estimates. First, collimation of the ejecta (jets) will result in overestimation of the total energy release. For GRB 990510 where a good case for a jet has been established (Figure 8), the standard isotropic energy estimate is probably a factor of 300 more than the true energy. ${ }^{47}$ Second, even after accounting for a possible jet geometry, the efficiency of converting the shock energy into gamma-ray emission is very uncertain. For example, some authors ${ }^{48}$ advocate low efficiency $(\sim 1 \%)$ which would result in an enormous upward correction to the usual isotropic estimates. Third, the bulk Lorentz factor is extremely high during the emission of $\gamma$-rays and thus the estimates critically depend on assumption of the geometry and granularity ${ }^{49}$ of the emitting region. In particular, if the emission is from small blobs ${ }^{49}$ then the inferred estimates are grossly in error.

In contrast to this highly uncertain situation, afterglows offer (in principle) more robust methods to evaluate $E_{0}$. In view of the importance of determining $E_{0}$ we summarize the different methods of determining $E_{0}$ from afterglow observations. One approach is to fit a "snapshot" broad-band afterglow spectrum (from radio to X-rays) to an afterglow model; this approach was pioneered by Wijers \& Galama. ${ }^{50}$ The strength of this method is that the estimated $E_{0}$ is, in principle, robust. Specifically, the estimate does not depend on the usually unknown environmental factors (run of density). However, in practice, this method is very sensitive to the values of the critical frequencies (Figure 7) which are usually not well determined. This difficulty explains the wildly differing estimates of $E_{0}$ for 
GRB 970508. ${ }^{50,51}$ Furthermore, this method uses measurements obtained at early times (when the afterglow at high frequencies is bright) with the result that the true source geometry is hidden by relativistic beaming.

A second approach is to model the light curves of the afterglow in a given band, specifically a radio band. The advantages of this method are the photometric stability of radio interferometers and the low Lorentz factor at the epoch of the peak of the radio emission. The disadvantages are two-fold: the sensitivity to the environmental parameters (density) and the assumption of the constancy of the microphysics parameters (electron and magnetic field equipartition factors). Application of this approach to GRB 980703 has resulted in seemingly accurate measures of the fireball parameters. ${ }^{33}$

Freedman \& Waxman ${ }^{52}$ take yet another approach, and estimate the energy release from late time X-ray observations. They show that the X-ray flux is insensitive to the GRB environment, and obtain robust estimates of the fireball energy per unit solid angle: from $3 \times 10^{51} \mathrm{erg}$ to $3 \times 10^{53} \mathrm{erg}$.

With all the above approaches, however, the possible collimation of the ejecta in jets is still a major uncertainty. This can be addressed by observing the evolution of the afterglow as the "edge" of the jet becomes visible. In most cases no evidence for jets has been seen, with the notable exceptions of GRB 990510 and possibly GRB 990123. In addition, a variety of statistical arguments (the absence of copious numbers of "orphan afterglows") ${ }^{53-55}$ suggests that, on average, the collimation cannot be extreme, and that for most bursts the opening angle is not less than 0.1 radian. Thus the total energy for most bursts may be reduced to the range of $10^{50}$ erg to $3 \times 10^{51} \mathrm{erg}$, but could easily be much higher in at least some cases.

Possibly the best approach to determining the energetics, which minimizes uncertainties due both to collimation (jets) and to the environment is to model the afterglow after it becomes non-relativistic. This method builds on the well established minimum energy formulation and the self-similarity of the Sedov solution. Not only are the ejecta truly non-relativistic, but they are also essentially spherical, as by this time jets will have had sufficient time to have undergone significant lateral expansion. Indeed, we can justifiably call this "fireball calorimetry". ${ }^{56}$ Applying this technique to the long-lived afterglow of GRB 970508 (Figure 1) led to the surprising result that $E_{0} \sim 5 \times 10^{50}$ erg weaker than a standard SN! This is an astonishing result. If true, this result would suggest that it is not $E_{0}$ which is the prime distinction between GRBs and $\mathrm{SNe}$ but the ejecta mass. However, Chevalier $\& \mathrm{Li}^{57}$ interpret the same data in the wind framework and derive much larger $E_{0}$. Clearly, we need more well studied afterglows with sufficient observations to first distinguish the circumburst environment (wind versus ISM) and then radio observations over a sufficiently long baseline to undertake calorimetry. Nonetheless, one should bear in mind that the current evidence for large energy release in GRBs is not as strong as is usually assumed.

\section{THE FUTURE}

It is generally accepted that GRB astronomy is an emerging and vibrant area of research in astronomy. We end this review with a brief discussion of potential progress and promise of this field.

The best way to foretell the future is to look for analogous situations in the past. In $\S 2$ we already discussed the parallels between the SN field and the GRB field. Here we discuss the numerous parallels with quasar astronomy. First discovered at radio wavelengths, we now study quasars across the electromagnetic spectrum. Although still identified by their gamma-ray properties, we now recognize the tremendous value of pan-chromatic GRB and afterglow studies. In both cases, there was considerable controversy about the distance scale. However, once this issue was settled, it became clear that quasars are the most energetic objects (sustained power) whereas GRBs are the most brilliant. For both, the ultimate energy appears to be related to black holes (albeit of different masses).

The raging issues in GRB astronomy today are the same that fueled quasar studies in the 1960's: the spatial distribution, the extraction of energy from the central engine, the transfer of energy from stellar scales to parsec scales, and the geometry of the relativistic outflow (sphere or jet). Astronomers took decades to unify the seemingly diverse types of quasars, and to conclude that there are two types of central engines: radio loud and radio quiet. Likewise, there may well be two types of GRB engines: rapidly and slowly spinning black holes emerging respectively from collapse of a rotating core of a massive star or coalescence of compact objects and the collapse of a massive star. This picture could potentially explain both the cosmologically located GRBs and SN $1998 \mathrm{bw}$.

The promises of this field are three-fold. First is the potential connection of GRBs with spinning black holes (discussed above). GRBs may well be nature's laboratary for Strong Gravity. Second, is the potential application of GRBs to study IGM. One expects a roll-off in quasars at redshifts around 5 or so simply because it does take some 
time to assemble a massive blackhole. In contrast, if GRBs come from massive stars then one can expect GRBs within tens of millions years of the birth of the first stars. Thus the expectation is that the IGM in the redshift range beyond 5 or so can be probed by GRBs. One can envisage a coordinated approach in the future: a GRB sentinel satellite in the sky hooked up to a 5-m telescope, ready to swing into action within tens of seconds of the detection of a GRB! Finally, the SN-GRB connection is a fundamental step in stellar evolution and with that perhaps we would have completed our understanding of the death of stars of all masses.

Acknowledgments. Our research is supported by NASA and NSF. JSB thanks the Fannie \& John Hertz Foundation for their generous support, AD holds a Millikan Postdoctoral Fellowship in Experimental Physics, TJG holds a Fairchild Foundation Postdoctoral Fellowship in Observational Astronomy and RS holds Fairchild Foundation Senior Fellowship in Theoretical Astrophysics. The VLA is a facility of the National Science Foundation operated under cooperative agreement by Associated Universities, Inc. The W. M. Keck Observatory is operated by the California Association for Research in Astronomy, a scientific partnership among California Institute of Technology, the University of California and the National Aeronautics and Space Administration. It was made possible by the generous financial support of the W. M. Keck Foundation.

\section{REFERENCES}

1. G. J. Fishman and C. A. Meegan, "Gamma-ray bursts," Ann. Rev. Astr. Ap. 33, pp. 415-458, 1995.

2. B. Paczyński and J. Rhoads, "Radio transients from gamma-ray bursts," ApJ 418, p. L5, 1993.

3. J. I. Katz, "Two populations and models of gamma-ray bursts," ApJ 422, pp. 248-259, 1994.

4. D. A. Frail and S. R. Kulkarni, "Radio counterpart searches of gamma-ray bursts," Astrophys. Space Sci. 231, pp. 277-280, 1995.

5. P. Mészáros and M. J. Rees, "Optical and long-wavelength afterglow from gamma-ray bursts," ApJ 476, pp. 232$237,1997$.

6. E. Waxman, "Gamma-ray burst afterglow: Confirming the cosmological fireball model," ApJ 489, pp. L33-L36, 1997.

7. G. Boella, R. C. Butler, G. C. Perola, L. Piro, L. Scarsi, and J. A. M. Bleeker, "Bepposax, the wide band mission for x-ray astronomy," A\&AS 122, pp. 299-307, 1997.

8. E. Costa, F. Frontera, J. Heise, M. Feroci, J. In'T Zand, F. Fiore, M. N. Cinti, D. Dal Fiume, L. Nicastro, M. Orlandini, E. Palazzi, M. Rapisarda, G. Zavattini, R. Jager, A. Parmar, A. Owens, S. Molendi, G. Cusumano, M. C. Maccarone, S. Giarrusso, A. Coletta, L. A. Antonelli, P. Giommi, J. M. Muller, L. Piro, and R. C. Butler, "Discovery of an x-ray afterglow associated with the gamma-ray burst of 28 february 1997.," Nature 387, pp. 783-785, 1997.

9. J. van Paradijs, P. J. Groot, T. Galama, C. Kouveliotou, R. G. Strom, J. Telting, R. G. M. Rutten, G. J. Fishman, C. A. Meegan, M. Pettini, J. Bloom, and H. Pedersen, "Transient optical emission from the error box of the $\gamma$-ray burst of 28 february 1997," Nature 386, pp. 686-689, Jan. 1997.

10. D. A. Frail, S. R. Kulkarni, S. R. Nicastro, M. Feroci, and G. B. Taylor, "The radio afterglow from the gamma-ray burst of 8 may 1997.," Nature 389, pp. 261-263, 1997.

11. M. R. Metzger, S. G. Djorgovski, S. R. Kulkarni, C. C. Steidel, K. L. Adelberger, D. A. Frail, E. Costa, and F. Fronterra, "Spectral constraints on the redshift of the optical counterpart to the $\gamma$-ray burst of 8 may 1997," Nature 387, p. 879, 1997.

12. S. R. Kulkarni, S. G. Djorgoski, A. N. Ramaprakash, R. Goodrich, J. S. Bloom, K. L. Adelberger, T. Kundic, L. Lubin, D. A. Frail, F. Frontera, M. Feroci, L. Nicastro, A. J. Barth, M. Davis, A. V. Filippenko, and J. Newman, "Identification of a host galaxy at redshift $\mathrm{z}=3.42$ for the gamma-ray burst of 14 december 1997.," Nature 393, pp. 35-39, 1998.

13. S. G. Djorgovski, S. R. Kulkarni, J. S. Bloom, R. Goodrich, D. A. Frail, L. Piro, and E. Palazzi, "Spectroscopy of the host galaxy of the gamma-ray burst 980703," ApJ 508, pp. L17-L20, Nov. 1998.

14. A. S. Fruchter, E. Pian, S. E. Thorsett, L. E. Bergeron, R. A. Gonzalez, M. Metzger, P. Goudfrooij, K. C. Sahu, H. Ferguson, M. Livio, M. Mutchler, L. Petro, F. Frontera, T. Galama, P. Groot, R. Hook, C. Kouveliotou, D. Macchetto, J. Van Paradijs, E. Palazzi, H. Pedersen, W. Sparks, and M. Tavani, "The fading optical counterpart of GRB 970228, 6 months and 1 year later," ApJ 516, pp. 683-692, May 1999.

15. S. Holland and J. Hjorth, "Star-forming regions near GRB 990123," A\&A 344, pp. L67-L70, 1999. 
16. J. S. Bloom, S. C. Odewahn, S. G. Djorgovski, S. R. Kulkarni, F. A. Harrison, C. Koresko, G. Neugebauer, L. Armus, D. A. Frail, R. R. Gal, R. Sari, G. Squires, G. Illingworth, D. Kelson, F. H. Chaffee, R. Goodrich, M. Feroci, E. Costa, L. Piro, F. Frontera, S. Mao, C. Akerlof, and T. A. McKay, "The host galaxy of GRB 990123," ApJ 518, pp. L1-L4, June 1999.

17. J. S. Bloom et al., "The unusual afterglow of GRB 980326: evidence for the gamma-ray burst/supernova connection," Nature 401, pp. 453-456, 1999.

18. T. J. Galama, P. M. Vreeswijk, J. Van Paradijs, C. Kouveliotou, T. Augusteijn, H. Bohnhardt, J. P. Brewer, V. Doublier, J.-F. Gonzalez, B. Leibundgut, C. Lidman, O. R. Hainaut, F. Patat, J. Heise, J. In'T Zand, K. Hurley, P. J. Groot, R. G. Strom, P. A. Mazzali, K. Iwamoto, K. Nomoto, H. Umeda, T. Nakamura, T. R. Young, T. Suzuki, T. Shigeyama, T. Koshut, M. Kippen, C. Robinson, P. De Wildt, R. A. M. J. Wijers, N. Tanvir, J. Greiner, E. Pian, E. Palazzi, F. Frontera, N. Masetti, L. Nicastro, M. Feroci, E. Costa, L. Piro, B. A. Peterson, C. Tinney, B. Boyle, R. Cannon, R. Stathakis, E. Sadler, M. C. Begam, and P. Ianna, "An unusual supernova in the error box of the gamma-ray burst of 25 april 1998.," Nature 395, pp. 670-672, 1998.

19. S. R. Kulkarni, D. A. Frail, M. H. Wieringa, R. D. Ekers, E. M. Sadler, R. M. Wark, J. L. Higdon, E. S. Phinney, and J. S. Bloom, "Radio emission from the unusual supernova $1998 \mathrm{bw}$ and its association with the gamma-ray burst of 25 April 1998," Nature 395, pp. 663-669, 1998.

20. S. R. Kulkarni, E. Berger, J. S. Bloom, F. Chaffee, A. Diercks, S. G. Djorgovski, D. A. Frail, T. J. Galama, R. W. Goodrich, F. A. Harrison, R. Sari, and S. A. Yost, "The afterglows of gamma-ray bursts." 5th Huntsville Gamma-Ray Burst Symposium, in press, astro-ph/0002168, 2000.

21. J. S. Bloom, S. G. Djorgovski, S. R. Kulkarni, and D. A. Frail, "The host galaxy of GRB 970508," ApJ 507, pp. L25-L28, 1998.

22. S. G. Djorgovski et al. "The radio afterglow from GRB 970828 and optical spectroscopy of its host galaxy." in preparation, 2000.

23. S. R. Kulkarni, S. G. Djorgovski, S. C. Odewahn, J. S. Bloom, R. R. Gal, C. D. Koresko, F. A. Harrison, L. M. Lubin, L. Armus, R. Sari, G. D. Illingworth, D. D. Kelson, D. K. Magee, P. G. Van Dokkum, D. A. Frail, J. S. Mulchaey, M. A. Malkan, I. S. MCClean, H. I. Teplitz, D. Koerner, D. Kirkpatrick, N. Kobayashi, I. A. Yadigaroglu, J. Halpern, T. Piran, R. W. Goodrich, F. H. Chaffee, M. Feroci, and E. Costa, "The afterglow, redshift, and extreme energetics of the gamma-ray burst of 23 January 1999," Nature 398, pp. 389-394, 1999.

24. D. Eichler, M. Livio, T. Firan, and D. N. Schramm, "Nucleosynthesis, neutrino bursts and gamma-rays from coalescing neutron stars," Nature 340, pp. 126-128, 1989.

25. J. M. Lattimer and D. N. Schramm, "Black-hole-neutron-star collisions," ApJ 192, pp. L145-L147, 1974.

26. R. Mochkovitch, M. Hernanz, J. Isern, and X. Martin, "Gamma-ray bursts as collimated jets from neutron star/black hole mergers," Nature 361, pp. 236-238, 1993.

27. R. Narayan, B. Paczyński, and T. Piran, "Gamma-ray bursts as the death throes of massive binary stars," $A p J$ 395, p. L83, 1992.

28. S. E. Woosley, "Gamma-ray bursts from stellar mass accretion disks around black holes," ApJ 405, pp. 273-277, 1993.

29. J. S. Bloom, S. Sigurdsson, and O. R. Pols, "The spatial distribution of coalescing neutron star binaries: implications for gamma-ray bursts," MNRAS 305, pp. 763-769, May 1999.

30. E. Pian, A. S. Fruchter, L. E. Bergeron, S. E. Thorsett, F. Frontera, M. Tavani, E. Costa, M. Feroci, J. Halpern, R. A. Lucas, L. Nicastro, E. Palazzi, L. Piro, W. Sparks, A. J. Castro-Tirado, T. Gull, K. Hurley, and H. Pedersen, "Hubble space telescope imaging of the optical transient associated with GRB 970508," ApJ 492, pp. L103L106, 1998.

31. S. G. Djorgovski, S. R. Kulkarni, J. S. Bloom, D. Frail, F. Chaffee, and R. Goodrich, "GRB 980613: Spectroscopy of the host galaxy." GCN notice 189, 1998.

32. A. S. Fruchter, S. E. Thorsett, M. R. Metzger, K. C. Sahu, L. Petro, M. Livio, H. Ferguson, E. Pian, D. W. Hogg, T. Galama, T. R. Gull, C. Kouveliotou, D. Macchetto, J. Van Paradijs, H. Pedersen, and A. Smette, "Hubble space telescope and palomar imaging of GRB 990123: Implications for the nature of gamma-ray bursts and their hosts," ApJ 519, pp. L13-L16, July 1999.

33. D. Frail, J. Bloom, S. Kulkarni, R. Sari, and G. Taylor, "The light curve and the spectrum of the radio afterglow of GRB 980703." in preparation, 2000.

34. T. J. Galama et al., "Evidence for a supernova in reanalyzed optical and near-infrared images of GRB 970228." submitted, astro-ph/9907264, 1999. 
35. D. E. Reichart, "GRB 970228 revisited: Evidence for a supernova in the light curve and late spectral energy distribution of the afterglow," ApJ 521, pp. L111-L115, Aug. 1999.

36. E. Waxman and B. T. Draine, "Dust sublimation by grbs and its implications." ApJ submitted; astroph/9909020, 1999.

37. P. M. Garnavich, R. P. Kirshner, P. Challis, J. Tonry, R. L. Gilliland, R. C. Smith, A. Clocchiatti, A. Diercks, A. V. Filippenko, M. Hamuy, C. J. Hogan, B. Leibundgut, M. M. Phillips, D. Reiss, A. G. Riess, B. P. Schmidt, R. A. Schommer, J. Spyromilio, C. Stubbs, N. B. Suntzeff, and L. Wells, "Constraints on cosmological models from hubble space telescope observations of high-z supernovae," ApJ 493, pp. L53-+, 1998.

38. J. I. Katz and L. M. Canel, "Long and short GRBs," in Gamma Ray Bursts: 3rd Huntsville Symposium, C. Kouveliotou, M. F. Briggs, and G. J. Fishman, eds., pp. 759-763, AIP, (Woodbury, New York), 1996.

39. L.-X. Li and B. Paczynski, "Transient events from neutron star mergers," ApJ 507, pp. L59-L62, 1998.

40. J. S. Bloom, S. R. Kulkarni, F. Harrison, T. Prince, E. S. Phinney, and D. A. Frail, "Expected characteristics of the subclass of supernova gamma-ray bursts," ApJ 506, pp. L105-L108, Oct. 1998.

41. R. Sari, T. Piran, and R. Narayan, "Spectra and light curves of gamma-ray burst afterglows," ApJ 497, p. L17, 1998.

42. R. A. Chevalier and Z.-Y. Li, "Gamma-ray burst environments and progenitors," ApJ 520, pp. L29-L32, July 1999.

43. J. C. Wheeler, "Cosmic explosions: Rapporteur summary of the 10th maryland astrophysics conference." in press, astro-ph/9912403, 1999.

44. R. Sari, T. Piran, and J. P. Halpern, "Jets in gamma-ray bursts," ApJ 519, pp. L17-L20, 1999.

45. D. Frail, S. Kulkarni, R. Sari, G. Taylor, D. Shepherd, J. Bloom, C. Young, L. Nicastro, and N. Masetti, "The radio afterglow from GRB 980519: A test of the jet and circumstellar models." ApJ in press; astro-ph/9910060, 1999.

46. D. A. Frail, S. R. Kulkarni, C. H. Young, and G. B. Taylor, "GRB 980329: A gamma-ray burst in a wind-blown medium." ApJ submitted, 2000.

47. F. A. Harrison, J. S. Bloom, D. A. Frail, R. Sari, S. R. Kulkarni, S. G. Djorgovski, T. Axelrod, J. Mould, B. P. Schmidt, M. H. Wieringa, R. M. Wark, R. Subrahmanyan, D. McConnell, P. J. McCarthy, B. E. Schaefer, R. G. McMahon, R. O. Markze, E. Firth, P. Soffitta, and L. Amati, "Optical and radio observations of the afterglow from GRB 990510: Evidence for a jet," ApJ 523, pp. L121-L124, 1999.

48. P. Kumar, "The distribution of burst energy and shock parameters for gamma-ray bursts." ApJ in press; astroph/9912566, 1999.

49. T. Piran and P. Kumar, "Energetics and luminosity function of gamma-ray bursts." ApJ in press; astroph/9909014, 1999.

50. R. A. M. J. Wijers and T. J. Galama, "Physical parameters of GRB 970508 and GRB 971214 from their afterglow synchrotron emission," ApJ 523, pp. 177-186, Sept. 1999.

51. J. Granot, T. Piran, and R. Sari, "Synchrotron self-absorption in gamma-ray burst afterglow," $A p J 527$, pp. 236246, 1999.

52. D. L. Freedman and E. Waxman, "On the energy of gamma-ray bursts." ApJ (Let) submitted;astro-ph/9912214, 1999.

53. J. E. Grindlay, "Fast x-ray transients and gamma-ray bursts: Constraints on beaming," ApJ 510, pp. 710-714, 1999.

54. J. Greiner, W. Voges, T. Boller, and D. Hartmann, "Search for GRB afterglows in the rosat all-sky survey," $A E A S$ 138, pp. 441-442, Sept. 1999.

55. J. E. Rhoads, "How to tell a jet from a balloon: A proposed test for beaming in gamma-ray bursts," ApJ $\mathbf{4 8 7}$ pp. L1-L4, 1997.

56. D. A. Frail, E. Waxman, and S. R. Kulkarni, "A 450-day light curve of the radio afterglow of GRB 970508: Fireball calorimetry." ApJ in press; astro-ph/9910319, 2000.

57. R. A. Chevalier and Z.-Y. Li, "Wind interaction models for gamma-ray burst afterglows: The case for two types of progenitors." ApJ (Let) submitted;astro-ph/9908272, 1999. 